\title{
MicroRNA-497 suppresses angiogenesis by targeting vascular endothelial growth factor A through the PI3K/AKT and MAPK/ERK pathways in ovarian cancer
}

\author{
WEI WANG ${ }^{1}$, FANG REN ${ }^{1}$, QINGHUA WU ${ }^{1}$, DAZHI JIANG ${ }^{2}$, HONGJUN LI ${ }^{2,3}$ and HUIRONG SHI ${ }^{1}$ \\ Departments of ${ }^{1}$ Obstetrics and Gynecology, and ${ }^{2}$ Nuclear Medicine, The First Affiliated Hospital \\ of Zhengzhou University, Zhengzhou, Henan 450052; ${ }^{3}$ Department of Radiology, \\ Beijing YouAn Hospital, Capital Medical University, Beijing 100054, P.R. China
}

Received June 16, 2014; Accepted August 5, 2014

DOI: $10.3892 /$ or.2014.3439

\begin{abstract}
MicroRNAs (miRNAs) have been shown to play an important role in diverse biological processes and cancer progression. The objective of the present study was to investigate the role of miR-497 in ovarian cancer angiogenesis. We found that miR-497 expression was downregulated in human ovarian cancer tissues, and the low miR-497 expression was significantly associated with increased angiogenesis. Functionally, exogenous expression of miR-497 suppressed the ability of ovarian cancer cells to promote capillary tube formation of endothelial cells. We further disclosed that miR-497 exerted its function of anti-angiogenesis by suppressing VEGFA expression in ovarian cancer cells and, in turn, impairing the VEGFR2-mediated PI3K/AKT and MAPK/ERK pathways. Our findings suggest that downregulation of miR-497 may contribute to angiogenesis in ovarian cancer. miR-497 may be a promising candidate target for prevention and treatment of ovarian cancer.
\end{abstract}

\section{Introduction}

Ovarian cancer is the leading cause of death from gynecological malignancies $(1,2)$. Active angiogenesis is essential for the tumor growth and metastasis of ovarian cancer, which in turn can lead to tumor recurrence and patient death $(3,4)$. Therefore, identifying molecules that can suppress angiogenesis may provide promising targets for ovarian cancer therapeutic strategies.

MicroRNAs (miRNAs), a class of short non-coding RNAs, are involved in the regulation of a variety of biological processes

Correspondence to: Dr Huirong Shi, Department of Obstetrics and Gynecology, The First Affiliated Hospital of Zhengzhou University, Zhengzhou, Henan 450052, P.R. China

E-mail: huirongshizzdx@163.com

Key words: ovarian carcinoma, microRNA, miR-497, angiogenesis, VEGFA, AKT, ERK
(5), including cellular metastasis (6) and angiogenesis $(7,8)$. miR-497, an important member of the miR-15/16/195/424/497 family of miRNAs (9), is dysregulated in various types of cancers, including ovarian cancer $(10,11)$. Previous studies suggest that miR-497 participates in various physiological and pathological processes, including cell proliferation (12), apoptosis (13), angiogenesis (14), migration $(15,16)$ and resistance to chemotherapy treatment (17). miR-497 has been reported to exert a tumor-suppressor function in human colorectal (16) and cervical cancer (18), adrenocortical carcinoma (19), neuroblastoma (13), primary plasma cell leukemia (20) and breast cancer (12). miR-497 was found to be downregulated in nonsmall cell lung cancer, and its ectopic expression significantly inhibited tumor growth and angiogenesis (14). However, its potential role in regulating ovarian carcinogenesis and angiogenesis is unclear.

Herein, we clarified the role of miR-497 in ovarian cancer angiogenesis by using in vitro assays and clinical ovarian cancer tissues. We found that downregulation of miR-497 in ovarian cancer tissues was associated with increased angiogenesis. Exogenous expression of miR-497 significantly suppressed ovarian cancer cell angiogenesis. We further provided evidence that miR-497 exerted its anti-angiogenic effect by targeting vascular endothelial growth factor A (VEGFA) via VEGFR2-mediated PI3K/AKT and MAPK/ ERK pathways. Our findings suggest that miR-497 may function as an anti-angiogenesis factor in ovarian cancer. miR-497 may be an important tumor suppressor and a future therapeutic target for ovarian cancer patients.

\section{Materials and methods}

Human tissue specimens. A total of 126 fresh-frozen human normal and malignant ovarian tissues were collected at the Department of Obstetrics and Gynecology of the First Affiliated Hospital of Zhengzhou University. Both tumor and normal ovarian tissues were histologically examined, and the tumor histology was serous cystadenocarcinoma. The tissue collection used for quantitative real-time PCR analysis included 30 normal ovarian tissue sections and 96 serous ovarian carcinoma tissues. Formalin-fixed, paraffin-embedded 
tissues, matching the frozen cases, were retrieved for immunohistochemical analysis. The patients had not received any local or systemic anticancer treatments prior to the surgery. The relevant clinical characteristics of the studied subjects are shown in Table I.

Informed consent was obtained from each patient, and the study was approved by the Institute Research Ethics Committee at the Cancer Center.

Cell lines and cell culture. The ovarian cancer cell lines OVCAR-3 and SK-OV-3 were purchased from the Institute of Biochemistry and Cell Biology of the Chinese Academy of Sciences (Shanghai, China). The cell lines were maintained in RMPI-1640 supplemented with $10 \%$ fetal bovine serum (FBS) (both from HyClone, USA), $100 \mathrm{U} / \mathrm{ml}$ of penicillin, and $100 \mathrm{~g} /$ $\mathrm{ml}$ of streptomycin at $37^{\circ} \mathrm{C}$ in $5 \% \mathrm{CO}_{2}$. Human umbilical vein endothelial cells (HUVECs) were purchased from the American Type Culture Collection (ATCC) and cultured in medium 199 (Gibco-Invitrogen, USA), supplemented with $20 \% \mathrm{FBS}, 50 \mathrm{mg} / \mathrm{ml}$ endothelial cell growth supplement (ECGS) (BD Biosciences, USA), $100 \mathrm{mg} / \mathrm{ml}$ heparin (Sigma, USA) and $1 \%$ penicilin-streptomycin. Cells were all cultured at $37^{\circ} \mathrm{C}$ in a $5 \% \mathrm{CO}_{2}$ incubator.

Lentivirus production and transfection. The human miR-497 precursor sequences were cloned into the lentivirus-based expression plasmid pLenti-6.3 (Invitrogen, USA). Viral packaging and infection were performed according to the standard protocols as recommended by the manufacturer. Cells were infected with $1 \times 10^{7}$ lentivirus transducing units in the presence of $10 \mu \mathrm{g} / \mathrm{ml}$ Polybrene (Sigma-Aldrich, St. Louis, MO, USA). An empty lentiviral vector was used as the negative control. The sequence-specific miR-497 inhibitor (anti-miR-497) and its control (anti-NC) were from BioCat GmbH (Heidelberg, Germany).

RNA isolation, reverse transcription and quantitative realtime PCR. Total RNA was extracted using TRIzol reagent (Invitrogen). To quantitate miR-497 expression, total RNA was polyadenylated and reversely transcribed using NCode miRNA First-Strand cDNA synthesis kit (Invitrogen). To measure the mRNA levels of VEGFA, total RNA was reversely transcribed using the PrimeScript RT reagent kit with gDNA Eraser (Takara, Japan). Quantitative real-time PCR was performed using SYBR Premix Ex Taq II (Takara) in Agilent Mx3005P. Primers used for miR-497 were: 5'-GTGCAGGGT CCGAGGT-3' (forward) and 5'-TAGCCTGCACACTGT GGT-3' (reverse). Primers used for VEGFA were: 5'-GTTTGA CAAGACCACCAAACT-3' (forward) and 5'-CCGCATAA TCTGCATGGTGAT-3' (reverse). GAPDH was used as an internal control. All samples were normalized to internal controls, and fold-changes were calculated through relative quantification $\left(2^{-\Delta \Delta C \mathrm{C}}\right)$.

Luciferase reporter assay. Double-stranded oligonucleotides corresponding to the wild-type (WT, 3'-UTR) or mutant (Mut, 3'-UTR) miR-497 binding site in the 3'-UTR of VEGFA were synthesized and subcloned into the GV272 reporter vector (GV272: SV40-Luciferase-MCS-PolyA was purchased from Shanghai GeneChem Co., Ltd.). Cells were transfected with
Table I. Correlation between miR-497 expression and clinicopathological features of the serous cystadenocarcinoma cases.

\begin{tabular}{|c|c|c|c|c|}
\hline \multirow{2}{*}{$\begin{array}{l}\text { Clinicopathological } \\
\text { features }\end{array}$} & \multirow{2}{*}{$\begin{array}{l}\text { Total } \\
\text { cases }\end{array}$} & \multicolumn{2}{|c|}{$\begin{array}{l}\text { miR-497 } \\
\text { expression }\end{array}$} & \multirow[b]{2}{*}{ P-value } \\
\hline & & High & Low & \\
\hline Group & & & & $0.0047^{\mathrm{b}}$ \\
\hline Normal tissue & 30 & 19 & 11 & \\
\hline Carcinoma tissue & 96 & 38 & 58 & \\
\hline Age (years) & & & & 0.144 \\
\hline$\leq 60$ & 39 & 20 & 19 & \\
\hline$>60$ & 57 & 18 & 39 & \\
\hline TNM stage & & & & $0.030^{\mathrm{b}}$ \\
\hline I-II & 31 & 11 & 20 & \\
\hline III-IV & 65 & 27 & 38 & \\
\hline Differentiation & & & & 0.130 \\
\hline Well & 21 & 9 & 12 & \\
\hline Moderate & 38 & 17 & 21 & \\
\hline Poor & 37 & 12 & 25 & \\
\hline Metastasis & & & & $0.040^{\mathrm{a}}$ \\
\hline Yes & 57 & 17 & 40 & \\
\hline No & 39 & 21 & 18 & \\
\hline Residual tumor size & & & & 0.718 \\
\hline Yes & 55 & 22 & 33 & \\
\hline No & 41 & 16 & 25 & \\
\hline
\end{tabular}

${ }^{a}$ Significant difference $(\mathrm{p}<0.05)$; ${ }^{\mathrm{b}}$ significant difference $(\mathrm{p}<0.01)$.

the appropriate plasmid and miR-497 duplex. Luciferase assays were carried out using the Luciferase reporter assay system (Promega, USA) $48 \mathrm{~h}$ after transfection. Normalized luciferase activity was reported as luciferase activity/Renilla luciferase activity.

Preparation of tumor-cell conditioned medium (TCM). Transfected cells were cultured in serum-free medium (SFM) for endothelial cells for $12 \mathrm{~h}$. The TCM was centrifuged sequentially at $500 \mathrm{x}$ g to remove the detached cells and at $12,000 \mathrm{x}$ g to discard cell debris $\left(4^{\circ} \mathrm{C}, 10 \mathrm{~min}\right.$ each). Aliquots of TCM were stored at $-80^{\circ} \mathrm{C}$ until used.

Capillary tube formation assay. HUVECs $\left(2.5 \times 10^{4}\right)$ were grown in the absence or presence of $100 \%$ TCM for $10 \mathrm{~h}$ at $37^{\circ} \mathrm{C}$ in a 96-well plate coated with Matrigel (3432-005-01; R\&D Systems, Minneapolis, MN, USA). The formation of capillary-like structures was captured under a light microscope. The branch points of the formed tubes, which represent the degree of angiogenesis in vitro, were scanned and quantitated in five low-power fields.

Western blot assay. For each sample, total protein extracts were separated on SDS-PAGE gels and transferred to nitrocellulose membranes, which were blocked with 5\% non-fat dry 
A

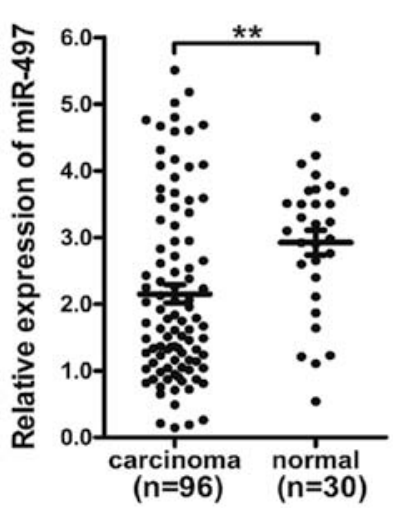

B

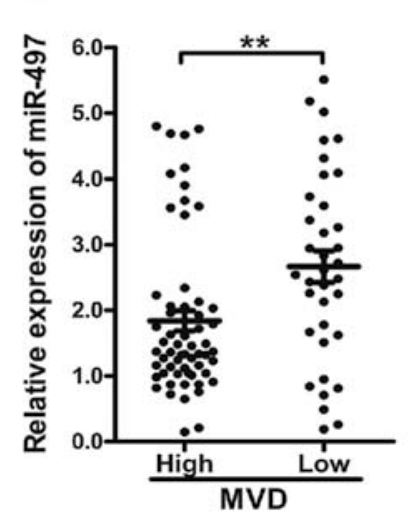

C

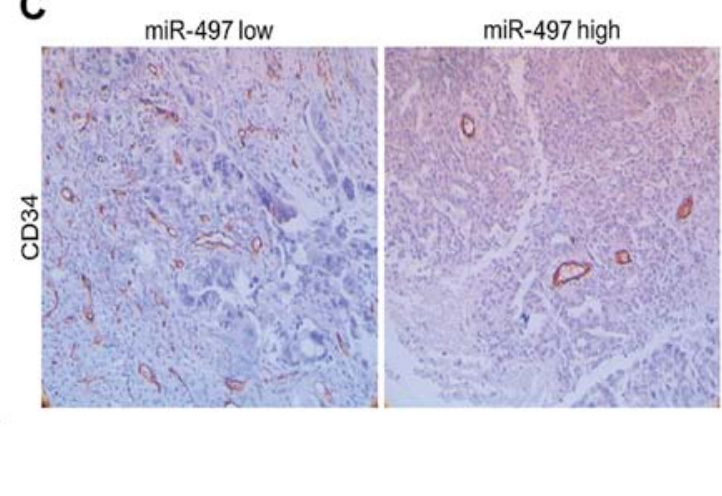

Figure 1. Expression of miR-497 in human ovarian cancer tissues. (A) The expression of miR-497 was measured by real-time qPCR in the human ovarian cancer tissues $(n=96)$ and normal ovarian tissues $(n=30)$. (B and C) Microvessel density (MVD) was inversely correlated with miR-497 expression in the human ovarian cancer tissues. The central horizontal line represents the mean value; ${ }^{* *} \mathrm{p}<0.01$.

milk for $2 \mathrm{~h}$ and incubated with antibodies. The antibodies for VEGFA, VEGFR2 and ERK1/ERK2 were obtained from LifeSpan BioSciences Inc. The antibodies for FAK and SHP-1 were obtained from Cell Signaling Technology. The corresponding bands were quantified by image processing software, ImageJ (NIH, http://rsb.nih.gov/ij/).

Immunohistochemistry. The antibodies included rabbit antiVEGFA/VEGF polyclonal antibody (LifeSpan BioSciences, Inc.) and rabbit anti-CD34 polyclonal antibody (ZSGBBiotechnology, China). Immunodetection was performed with a Vectastain ABC kit (Vector Laboratories). The microvessel density (MVD) in tumor tissues, which represents the degree of angiogenesis in vivo, was evaluated by staining for CD34, an endothelial cell marker. Any discrete cluster or single cell stained for CD34 was counted as one microvessel.

Statistical analysis. The data are expressed as the means \pm standard error of the mean (SEM) from at least three independent experiments. The values for the capillary tube formation and luciferase activity assays are from three independent experiments that were performed in duplicate. The differences between the groups were analyzed by the Student's t-test when two groups were compared or by one-way ANOVA when more than two groups were compared. Analyses were performed with GraphPad Prism, version 5 (GraphPad Software, Inc., San Diego, CA, USA). Correlations between two variables were explored with the Spearman's correlation coefficient. All statistical tests were two-sided, and $\mathrm{p}<0.05$ was considered to indicate a statistically significant result.

\section{Results}

Downregulation of miR-497 in ovarian cancer tissues is associated with increased angiogenesis. To assess the clinical importance of miR-497 in ovarian cancer, we examined miR-497 levels in ovarian cancer tissues and normal ovarian tissues by real-time PCR. As shown in Fig. 1A, the expression levels of miR-497 in the ovarian cancer tissues were much lower than those in the normal ovarian tissues $(p=0.0047)$. Furthermore, lower miR-497 levels were associated with higher MVDs ( $p=0.0032$; Fig. 1B and C), suggesting that miR-497 downregulation may contribute to ovarian cancer progression by promoting tumor angiogenesis.

miR-497 inhibits ovarian cancer cell angiogenesis. To explore the effect of miR-497 on ovarian cancer angiogenesis, an in vitro capillary tube formation assay was carried out in SK-OV-3 and OVCAR-3 cells. We found that, compared with the control media (SFM), TCM from the negative control (NC)-transfected and non-transfected cells promoted the ability of HUVECs to develop more capillary-like structures (Fig. 2A and B). Tube formation was reduced dramatically in the HUVECs that were grown in TCM from the miR497-transfectants, to a level comparable to that of the HUVECs cultured in SFM (Fig. 2A and B). In contrast, the suppression of endogenous miR-497 in ovarian cancer cells resulted in enhanced capillary tube formation (Fig. 2C and D).

miR-497 inhibits ovarian cancer angiogenesis by directly targeting VEGFA. We further investigated the molecular mechanism by which miR-497 inhibits ovarian cancer angiogenesis. Putative targets of miR-497 were predicted with TargetScan. Among these, VEGFA was selected for further research due to its well-known importance in tumor angiogenesis. Dualluciferase reporter assay revealed that the co-transfection of miR-497 significantly inhibited the activity of firefly luciferase reporter with wild-type 3'UTR of VEGFA, whereas this effect was abrogated when the predicted 3'UTR binding site was mutated (Fig. 3A and B). Western blot analysis showed that the ectopic expression of miR-497 in the SK-OV-3 and OVCAR-3 cells resulted in the downregulation of VEGFA at the protein level (Fig. 3C and E). To confirm the effects of miR-497 on VEGFA, we transferred anti-miR-497 into ovarian cancer cells and found that inhibition of miR-497 increased the protein level of VEGFA (Fig. 3D and F). Moreover, an inverse correlation between miR-497 and VEGFA expression was confirmed in the human ovarian cancer tissues (Fig. 3G and H). These data indicate that miR-497 may negatively regulate VEGFA expression by directly targeting its 3'UTR.

We further validated that VEGFA mediates the anti-angiogenic function of miR-497. Suppression of VEGFA in ovarian 
A
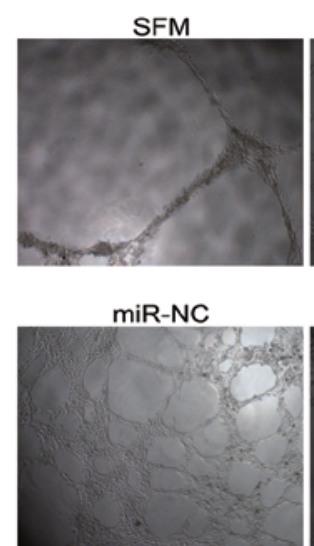

B

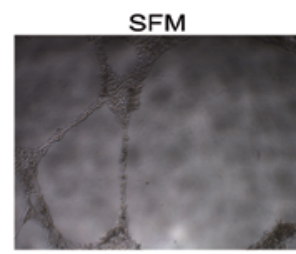

miR-NC

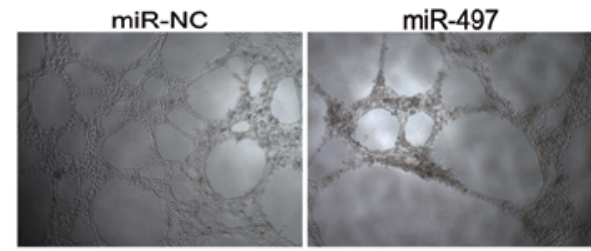

SK-OV-3

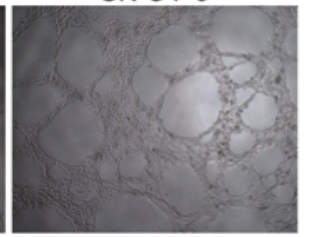

$\operatorname{miR}-497$

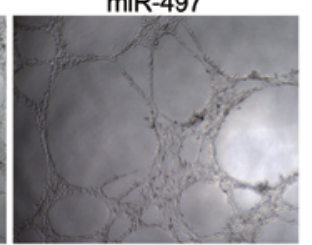

OVCAR-3

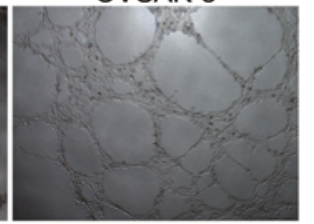

$\operatorname{miR}-497$

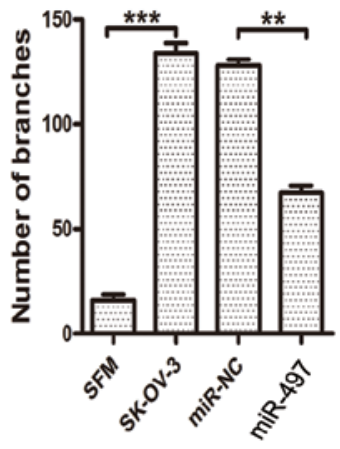

C

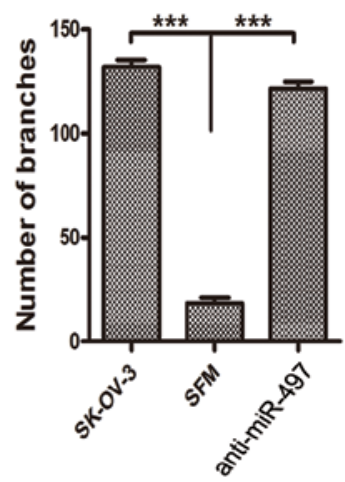

D
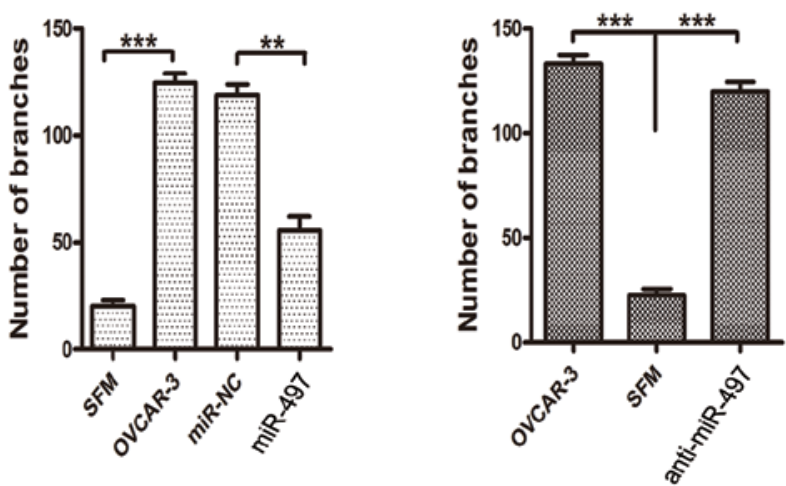

Figure 2. miR-497 suppresses ovarian cancer cell angiogenesis. (A and B) Restoration of miR-497 inhibited ovarian cancer cell-promoted EC tube formation. (C and D) Antagonism of miR-497 increased ovarian cancer cell-promoted EC tube formation. miR-NC, non-specific control miRNA; SFM, serum-free medium. ${ }^{* *} \mathrm{p}<0.01 ;{ }^{* * *} \mathrm{p}<0.001$.

cancer cells significantly reduced capillary tube formation (Fig. 4A and B). In contrast, overexpression of VEGFA in the miR-497-transfected cells attenuated the anti-angiogenic effects of miR-497 (Fig. 4D). In addition, human ovarian cancer tissues with higher MVD exhibited higher levels of VEGFA ( $p=0.0060$, Fig. 4C), corresponding to the correlation between lower miR-497 expression and higher MVD/VEGFA levels in ovarian cancer tissues (Figs. 1B and 3G).

miR-497 indirectly regulates the VEGFR2-mediated $P I 3 K / A K T$ and MAPK/ERK pathways by targeting VEGFA. VEGFR2 is the major receptor of VEGFA that mediates angiogenic activity, and the VEGF/VEGFR2 signaling pathway plays a crucial role in angiogenesis (21). To ascertain whether miR-497 affects expression of VEGFA through this signaling pathway, we determined the effects of miR-497 on the activation of VEGFR2 by immunoblotting of phospho-VEGFR2. As shown in Fig. 4, the miR-497-transfected ovarian cancer cells displayed significantly decreased phosphorylation of VEGFR2 when compared with the negative control cells.

To further understand how miR-497 exerts its anti-angiogenic effects via VEGFR2-mediated signaling, several key VEGFR2 downstream signaling molecules were studied. Western blot assay revealed that compared to the negative control cells, the miR-497-transfected ovarian cancer cells had significantly decreased phosphorylation of AKT and ERK1/2, while the phosphorylation status of other kinases such as SHP-1 and FAK were not significantly affected (Fig. 4E). In contrast, anti-miR-497-transfectants enhanced PI3K/AKT and MAPK/ERK signaling in the ovarian cancer cells (Fig. 4E). These results suggest that miR-497 may inhibit angiogenesis by downregulation of VEGFA expression via the VEGFR2-mediated PI3K/AKT and MAPK/ERK pathways in ovarian cancer cells.

\section{Discussion}

Angiogenesis, the process of new blood vessel formation, is a critical component in tumorigenesis for promoting cancer cell growth, invasion and metastasis, which in turn can lead to tumor recurrence and patient death $(22,23)$. Previous reports have demonstrated that miRNAs are involved in the regulation of tumor angiogenesis (24). For instance, it has been reported that the anti-angiogenesis role of miR-503 in tumorigenesis is by downregulation of FGF2 and VEGFA in human hepatocellular carcinomas (25). miR-15a and miR-16 were found to affect the angiogenesis of multiple myeloma by targeting VEGF (26). miR-15b and miR-152 reduced glioma cell invasion and angiogenesis by targeting NRP-2 and MMP-3 (27). miR-145 inhibited tumor angiogenesis and growth by targeting p70S6K1, HIF-1 and VEGF in human colon cancer (28) and by targeting HIF-2 $\alpha$ in neuroblastoma (29). miR-19b-1 was found to target mRNA corresponding to the pro-angiogenic protein, FGFR 2 and to block the cell cycle by controlling the 
A
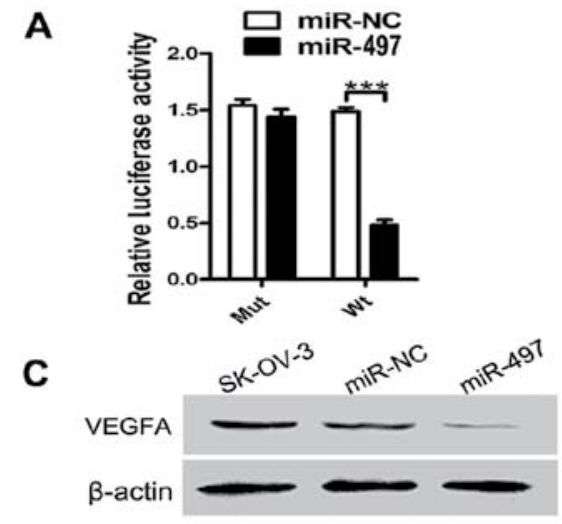

E

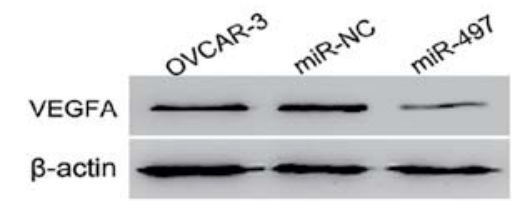

B

VEGFA

Wt 3' UTR CCAUUUUAUUUUUCUUGCUGCUA

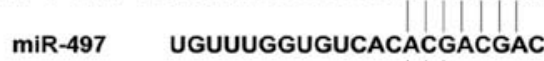

H.

Mut 3'UtR cCaUUUUaUUUUUCUUGCGUagc
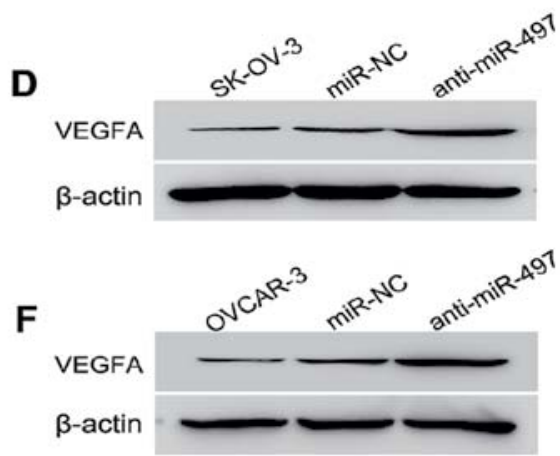

Relative miR-497 leve

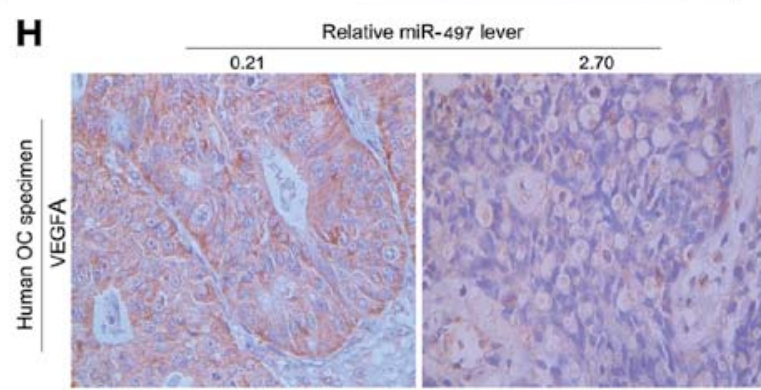

Figure 3. miR-497 inhibits VEGFA expression by targeting its 3'UTR. (A and B) Direct regulation of VEGFA by miR-497 through binding to the 3'UTR of VEGFA mRNA. (C and E) Western blot assay shows that expression of miR-497 reduced the protein levels of endogenous VEGFA in (C) SK-OV-3 and (E) OVCAR-3 cells. (D and F) Western blotting shows that depletion of endogenous miR-497 enhanced the cellular VEGFA protein level in (D) SK-OV-3 and (F) OVCAR-3 cells. miR-NC, non-specific control miRNA. (G and H) The levels of miR-497 are inversely correlated with VEGFA expression in 65 ovarian cancer tissues. VEGFA was also detected by immunohistochemical staining. Each data point represents an individual sample, and the correlation coefficient (r) is indicated; the central horizontal line represents the mean value; ${ }^{* * *} \mathrm{p}<0.001$. VEGFA, vascular endothelial growth factor A.

expression of cyclin D1 (30). Therefore, miRNAs that possess anti-angiogenic activities may provide novel targets for anticancer therapies.

The preliminary experiments revealed that miR-497 was downregulated in ovarian cancer, yet the function and the molecular mechanism of miR-497 in ovarian cancer still remained to be elucidated. Herein, we clarified that miR-497 functioned as a tumor suppressor in the angiogenesis of ovarian cancer. We found that miR-497 was downregulated in human ovarian cancer, and altered miR-497 expression was inversely associated with MVD in ovarian cancer tissues. Exogenous expression of miR-497 in the ovarian cancer cells suppressed tumor angiogenesis. These results indicate that downregulation of miR-497 may contribute to ovarian cancer progression by promoting tumor angiogenesis.

VEGFA, the most potent angiogenic factor, is responsible for tumor growth and angiogenesis $(31,32)$. Recently, a metaanalysis revealed that overexpression of VEGFA in primary tumors and serum is associated with poor progression-free survival and overall survival in patients with ovarian cancer (33). We found that VEGFA was negatively regulated by $\mathrm{miR}-497$. In the human ovarian cancer specimens, higher levels of miR-497 were closely correlated with lower expression of VEGFA, indicating that miR-497 may inhibit VEGFA expression in clinical ovarian cancer tissues. Exogenous expression of miR-497 in human ovarian cancer cell lines SK-OV-3 and OVCAR-3 inhibited VEGFA at the protein level, further confirming that miR-497 targets VEGFA protein. In addition, a positive correlation between MVD and VEGFA expression was observed in the clinical ovarian cancer specimens, corresponding to a correlation between lower miR-497 expression and higher MVD/VEGFA levels in ovarian cancer tissues. Owing to the specific expression profile of miR-497 in ovarian cancer that is consistent with other types of human cancers and the high expression of VEGFA that is strongly associated with ovarian cancer, miR-497 and VEGFA are useful biomarker for the clinical treatment of ovarian cancer.

VEGFR2 is a major receptor for VEGF. This receptor functions as the main mediator of VEGF-induced endothelial proliferation, tubular morphogenesis and sprouting (21). Multiple VEGFR2 downstream signaling mediators, including PI3K-AKT, ERK, SHP-1 and FAK are known to be involved in the regulation of endothelial cell proliferation and migration $(21,34)$. We found that reduction in VEGFA decreased tube formation in ovarian cancer cells and attenuated VEGFR2-mediated PI3K/AKT and MAPK/ERK pathway activation. On the other hand, overexpression of miR-497 had little effects on FAK and JNK phosphorylation. Our 
A

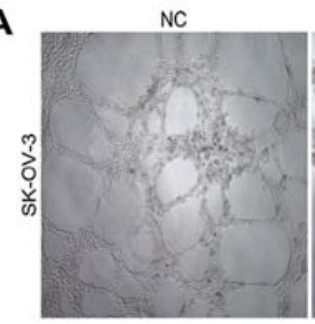

B

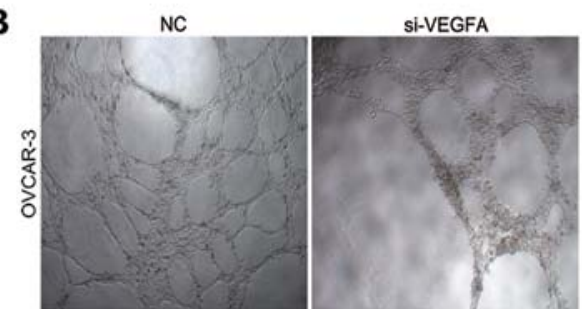

C

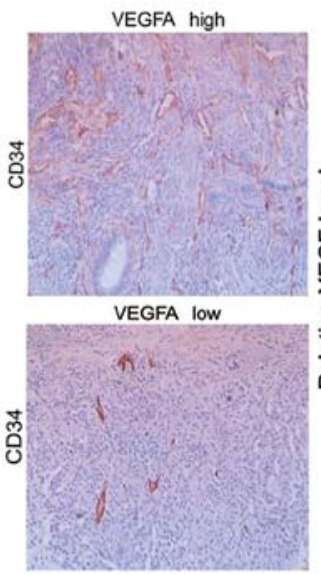

si-VEGFA

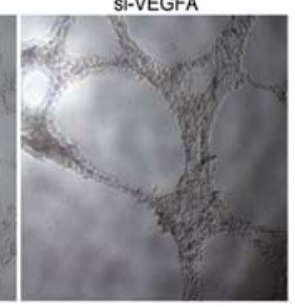

Si-VEGFA

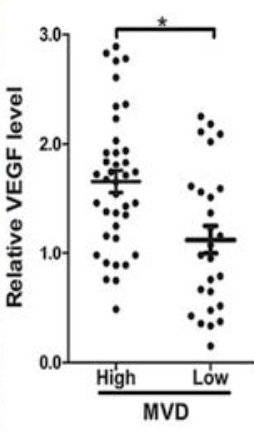

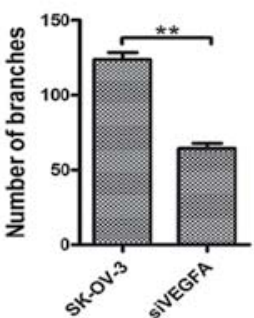

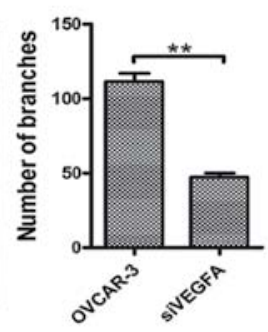

E
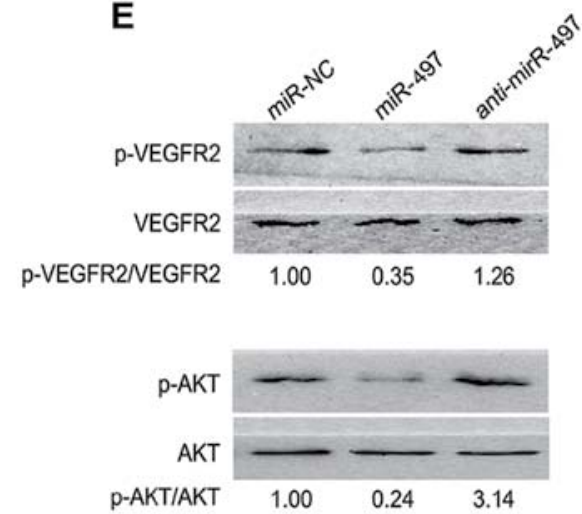

p-ERK1/2

ERK1/2

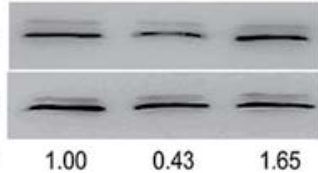

D

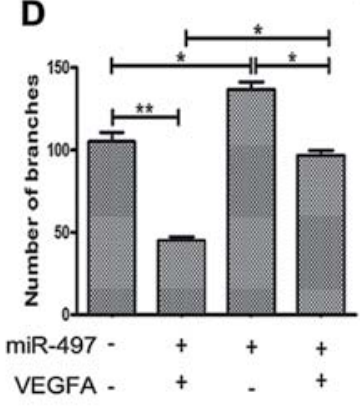

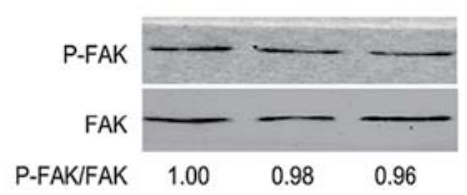

p-SHP-1

SHP-1

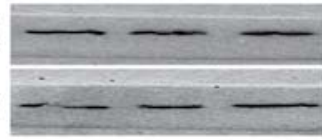

1.09

Figure 4. miR-497 suppresses VEGFA expression leading to reduced tumor cell angiogenic activity via the VEGFR2-mediated PI3K/AKT and MAPK/ERK pathways. (A and B) Suppression of VEGFA by RNAi reduces the ability of (A) SK-OV-3 and (B) OVCAR-3 cells to promote capillary tube formation. (C) Microvessel density (MVD) is positively correlated with VEGFA expression in ovarian cancer tissues. The median MVD value of the 65 cases that were used in Fig. 3G was chosen as the cut-off point for separating the low- $(n=26)$ and high-MVD $(n=39)$ groups. (D) Overexpression of VEGFA attenuated the anti-angiogenic effect of miR-497. (E) Effect of miR-497 expression on the VEGFR2-mediated PI3K/AKT and MAPK/ERK pathways in ovarian cancer cells. ${ }^{*} \mathrm{p}<0.05 ;{ }^{* *} \mathrm{p}<0.01$. VEGFA, vascular endothelial growth factor $\mathrm{A}$.

findings are in line with $\mathrm{Xu}$ et al (35) who found that miR148a and miR-152 inhibited breast cancer cell angiogenesis via targeting IGF-IR and IRS1 and suppressed their downstream AKT and MAPK/ERK signaling pathways. Da Silva et al (36) demonstrated that miR-126 is related to cardiac angiogenesis by indirect regulation of VEGF expression through the MAPK and PI3K/AKT/Enos pathways. Bao et al (37) found that miR-21 suppressed PTEN and hSult-1 expression and promoted hepatocellular carcinoma angiogenesis through AKT/ERK pathways. Our findings, along with other results, indicate that miR-497 downregulation in ovarian cancer cells may result in enhanced VEGF levels in the tumor microenvironment, which subsequently activate VEGFR2-mediate PI3K/AKT and MAPK/ERK signaling in endothelial cells and thereby promote angiogenesis.

Recently, several miRNAs have been reported to either promote or suppress ovarian cancer angiogenesis, including miR-378 (38), the miR-200 family (39), miR-484 (40) and miR-27a (41). These results combined with ours demonstrate that miR-497, as a tumor suppressor, could be considered as an anti-angiogenic factor for ovarian cancer. Our findings may be useful to better understand the mechanism of ovarian cancer development and identify miR-497 as a promising therapeutic target for ovarian cancer patients.

\section{Acknowledgements}

This study was supported by Grants from the National Natural Science Foundation of China (grant no. 81171986), and from the Basic and Advanced Technology Research Foundation of Henan Province (grant no. 112300410153).

\section{References}

1. Siegel R, Naishadham D and Jemal A: Cancer statistics, 2013. CA Cancer J Clin 63: 11-30, 2013.

2. Jemal A, Bray F, Center MM, Ferlay J, Ward E and Forman D: Global cancer statistics. CA Cancer J Clin 61: 69-90, 2011.

3. Weis SM and Cheresh DA: Tumor angiogenesis: molecular pathways and therapeutic targets. Nat Med 17: 1359-1370, 2011.

4. Carmeliet P and Jain RK: Molecular mechanisms and clinical applications of angiogenesis. Nature 473: 298-307, 2011.

5. Croce CM: miRNAs in the spotlight: understanding cancer gene dependency. Nat Med 17: 935-936, 2011. 
6. Su Y, Li X, Ji W, et al: Small molecule with big role: microRNAs in cancer metastatic microenvironments. Cancer Lett 344 : 147-156, 2014.

7. Eilken HM and Adams RH: Turning on the angiogenic microswitch. Nat Med 16: 853-854, 2010.

8. Conteduca V, Kopf B, Burgio SL, Bianchi E, Amadori D and De Giorgi U: The emerging role of anti-angiogenic therapy in ovarian cancer (Review). Int J Oncol 44: 1417-1424, 2014.

9. Finnerty JR, Wang WX, Hébert SS, Wilfred BR, Mao G and Nelson PT: The miR-15/107 group of microRNA genes: evolutionary biology, cellular functions, and roles in human diseases. J Mol Biol 402: 491-509, 2010.

10. Flavin RJ, Smyth PC, Laios A, et al: Potentially important microRNA cluster on chromosome $17 \mathrm{p} 13.1$ in primary peritoneal carcinoma. Mod Pathol 22: 197-205, 2009.

11. Zhang L, Huang J, Yang N, et al: microRNAs exhibit high frequency genomic alterations in human cancer. Proc Natl Acad Sci USA 103: 9136-9141, 2006.

12. Tahiri A, Leivonen SK, Lüders T, et al: Deregulation of cancerrelated miRNAs is a common event in both benign and malignant human breast tumors. Carcinogenesis 35: 76-85, 2014.

13. Creevey L, Ryan J, Harvey H, et al: MicroRNA-497 increases apoptosis in $M Y C N$ amplified neuroblastoma cells by targeting the key cell cycle regulator WEE1. Mol Cancer 12: 23, 2013.

14. Zhao WY, Wang Y, An ZJ, et al: Downregulation of miR-497 promotes tumor growth and angiogenesis by targeting HDGF in non-small cell lung cancer. Biochem Biophys Res Commun 435 466-471, 2013.

15. Li D, Zhao Y, Liu C, et al: Analysis of miR-195 and miR-497 expression, regulation and role in breast cancer. Clin Cancer Res 17: 1722-1730, 2011.

16. Guo ST, Jiang CC, Wang GP, et al: MicroRNA-497 targets insulin-like growth factor 1 receptor and has a tumour suppressive role in human colorectal cancer. Oncogene 32: 1910-1920, 2013.

17. Kim YW, Kim EY, Jeon D, et al: Differential microRNA expression signatures and cell type-specific association with Taxol resistance in ovarian cancer cells. Drug Des Devel Ther 8 : 293-314, 2014

18. Lajer CB, Garnæs E, Friis-Hansen L, et al: The role of miRNAs in human papilloma virus (HPV)-associated cancers: bridging between HPV-related head and neck cancer and cervical cancer Br J Cancer 106: 1526-1534, 2012.

19. Caramuta S, Lee L, Ozata DM, et al: Clinical and functional impact of TARBP2 over-expression in adrenocortical carcinoma. Endocr Relat Cancer 20: 551-564, 2013.

20. Lionetti M, Musto P, Di Martino MT, et al: Biological and clinical relevance of miRNA expression signatures in primary plasma cell leukemia. Clin Cancer Res 19: 3130-3142, 2013.

21. Claesson-Welsh L and Welsh M: VEGFA and tumour angiogenesis. J Intern Med 273: 114-127, 2013.

22. Welti J, Loges S, Dimmeler S and Carmeliet P: Recent molecular discoveries in angiogenesis and antiangiogenic therapies in cancer. J Clin Invest 123: 3190-3200, 2013.

23. Larsen AK, Ouaret D, El Ouadrani K and Petitprez A: Targeting EGFR and $\operatorname{VEGF}(\mathrm{R})$ pathway cross-talk in tumor survival and angiogenesis. Pharmacol Ther 131: 80-90, 2011.

24. Anand S and Cheresh DA: Emerging role of micro-RNAs in the regulation of angiogenesis. Genes Cancer 2: 1134-1138, 2011
25. Zhou B, Ma R, Si W, et al: MicroRNA-503 targets FGF2 and VEGFA and inhibits tumor angiogenesis and growth. Cancer Lett 333: 159-169, 2013.

26. Sun CY, She XM, Qin Y, et al: miR-15a and miR-16 affect the angiogenesis of multiple myeloma by targeting VEGF. Carcinogenesis 34: 426-435, 2013.

27. Zheng X, Chopp M, Lu Y, Buller B and Jiang F: MiR-15b and miR-152 reduce glioma cell invasion and angiogenesis via NRP-2 and MMP-3. Cancer Lett 329: 146-154, 2013.

28. Xu Q, Liu LZ, Qian X, et al: MiR-145 directly targets p70S6K1 in cancer cells to inhibit tumor growth and angiogenesis. Nucleic Acids Res 40: 761-774, 2012.

29. Zhang H, Pu J, Qi T, et al: MicroRNA-145 inhibits the growth, invasion, metastasis and angiogenesis of neuroblastoma cells through targeting hypoxia-inducible factor 2 alpha. Oncogene 33: 387-397, 2014

30. Yin R, Bao W, Xing Y, Xi T and Gou S: MiR-19b-1 inhibits angiogenesis by blocking cell cycle progression of endothelial cells. Biochem Biophys Res Commun 417: 771-776, 2012.

31. Saharinen P, Eklund L, Pulkki K, Bono P and Alitalo K: VEGF and angiopoietin signaling in tumor angiogenesis and metastasis. Trends Mol Med 17: 347-362, 2011.

32. Eng L, Azad AK, Habbous S, et al: Vascular endothelial growth factor pathway polymorphisms as prognostic and pharmacogenetic factors in cancer: a systematic review and meta-analysis. Clin Cancer Res 18: 4526-4537, 2012.

33. Yu L, Deng L, Li J, Zhang Y and Hu L: The prognostic value of vascular endothelial growth factor in ovarian cancer: a systematic review and meta-analysis. Gynecol Oncol 128: 391-396, 2013

34. Ferrara N: Pathways mediating VEGF-independent tumor angiogenesis. Cytokine Growth Factor Rev 21: 21-26, 2010.

35. Xu Q, Jiang Y, Yin Y, et al: A regulatory circuit of miR-148a/152 and DNMT1 in modulating cell transformation and tumor angiogenesis through IGF-IR and IRS1. J Mol Cell Biol 5: 3-13, 2013 .

36. DA Silva ND Jr, Fernandes T, Soci UP, Monteiro AW, Phillips MI and DE Oliveira EM: Swimming training in rats increases cardiac microRNA-126 expression and angiogenesis. Med Sci Sports Exerc 44: 1453-1462, 2012.

37. Bao L, Yan Y, Xu C, et al: MicroRNA-21 suppresses PTEN and hSulf-1 expression and promotes hepatocellular carcinoma progression through AKT/ERK pathways. Cancer Lett 337: 226-236, 2013

38. Chan JK, Kiet TK, Blansit K, et al: MiR-378 as a biomarker for response to anti-angiogenic treatment in ovarian cancer. Gynecol Oncol 133: 568-574, 2014

39. Pecot CV, Rupaimoole R, Yang D, et al: Tumour angiogenesis regulation by the miR-200 family. Nat Commun 4: 2427, 2013.

40. Vecchione A, Belletti B, Lovat F, et al: A microRNA signature defines chemoresistance in ovarian cancer through modulation of angiogenesis. Proc Natl Acad Sci USA 110: 9845-9850, 2013.

41. Lai Y, Zhang X, Zhang Z, et al: The microRNA-27a: ZBTB10specificity protein pathway is involved in follicle stimulating hormone-induced VEGF, Cox 2 and survivin expression in ovarian epithelial cancer cells. Int J Oncol 42: 776-784, 2013. 